\title{
LES OF THE FLOW AROUND TWO CYLINDERS IN TANDEM
}

\author{
Guillermo Palau-Salvador ${ }^{1}$, Thorsten Stoesser ${ }^{2}$, Jochen Fröhlich ${ }^{3}$, Wolfgang Rodi ${ }^{4}$ \\ ${ }^{1}$ Rural Engineering Dep., Polytechnic Univ. of Valencia, 46022, Valencia, Spain. guipasal@agf.upv.es \\ ${ }^{2}$ School of Civil and Environmental Eng. Georgia Inst. of Tech., Atlanta,US. thorsten@ce.gatech.edu \\ ${ }^{3}$ Institute of Fluid Mechanics. Technical University of Dresden. Jochen.Froehlich@tu-dresden.de \\ ${ }^{4}$ Institute for Hydromechanics, Karlsruhe University, 76128 Karlsruhe, Germany. rodi@ifh.uka.de
}

\begin{abstract}
The flow around an arrangement of two-in-tandem cylinders exhibits a remarkably complex behaviour that is of interest for many engineering problems, such as environmental flows or structural design. In the present paper, a Large Eddy Simulation using a staggered Cartesian grid has been performed for the flow around two-in-tandem cylinders of diameter $\mathrm{D}=20 \mathrm{~mm}$ and height $\mathrm{H}=50 \mathrm{~mm}$ submerged in an open channel with height $\mathrm{h}=60 \mathrm{~mm}$. The two axes have a streamwise spacing of $2 \mathrm{D}$. The Reynolds number is 1500 , based on the cylinder diameter and the free-stream velocity $\mathrm{u}_{\infty}$. The results obtained show that no vortex shedding occurs in the gap between the two cylinders where the separated shear layers produced by the upstream cylinder reattach on the surface of the downstream one. The flow separates on the top of the first cylinder with the presence of two spiral nodes known as owl-face configuration. On top of the downstream cylinder, the flow is attached. A complex mean flow develops in the gap and also behind the second cylinder. Comparisons with PIV measurements reveal good general agreement, but there are differences concerning some details of the flow in the gap between the cylinders.
\end{abstract}

Key words: LES, two-in-tandem cylinders, turbulence, fluid structure interaction.

\section{Introduction}

The flow around two cylinders (either of finite of infinite height) in tandem represents an important and remarkably complex flow configuration that has been the subject of experimental and numerical investigations for several decades (e.g. Igarashi [1], Zdravkovich [2]). This configuration has an important relevance for the study of flows around buildings [3], forces on bridge piers [4] or turbulence in vegetated open channels [5].

The interference that arises when more than one body is placed within the fluid stream is responsible for several changes in the characteristics of the flow. Depending on the distance between the cylinders, different flow regimes can occur and in the case of finite height cylinders mainly two-dimensional largescale structures are present. Classifications of qualitative flow regimes of the tandem cylinder arrangement can be found in Iragashi [1] and Zdravkovich [2]. Essentially, there is a critical value of $\mathrm{L} / \mathrm{D}$ ( $\mathrm{L}$ being the distance between the axes 
of the cylinders and D the diameter of each cylinder) below which no vortex shedding occurs in the gap region. At this value, the flow changes from a coshedding type, with both cylinders shedding vortices, to the reattached type. The latter is characterized by a shear layer that separates from the upstream cylinder and reattaches on the downstream cylinder [2]. According to the latter description, one can classify the flow around two-in-tandem cylinders in 4 types: single-slender-body regime, due to the proximity of the cylinders, the shear layer from the upstream cylinder rolls up after the downstream cylinder, forming a single wake; quasi-steady reattachment regime, the shear layer of the upstream cylinder reattaches on the downstream one; unstable region, vortex shedding behind the front cylinder occurs, but is intermittently suppressed and replaced by the reattachment flow regime; and the co-shedding region, where vortex shedding occurs past both cylinders. The critical value and the appearance of each flow regime depend on the Reynolds number as the experiments by Zdravkodich [2] and Ljungkrona et al. [6] have shown.

When the cylinders are of finite height, additional trailing vortices originating at the free end of the cylinders come into play adding further complexity to the flow. The tandem arrangement can be viewed as a simplification of an array of a large number of cylinders, and some of the characteristics of the tandem flow should be present, at least to some degree, also in an array.

Several qualitative flow-visualizations of the gap and near-wake flow patterns are reported in the literature. Oka et al. [7] measured vortex-shedding frequencies; they did not observe any shedding until a gap distance greater than 3.8 diameters. Other authors found similar values: Tanida et al [8] obtained a value of 3 diameters and Igarashi [1] of 3.53 diameters. However, other authors, like Ljungkrona et al. [6] clearly show the important influence of the Reynolds number on the critical value of $\mathrm{L} / \mathrm{D}$ ( 4.5 for $\mathrm{Re}=1400$ and 3.0 for $\mathrm{Re}=42000$ ). Sumner et al [9] and Kadota et al. [4] used Particle Imaging Velocimeter to measure the flow in the gap region of the two in tandem cylinders.

Apart from the above experimental studies, very few numerical simulations exist which furthermore are mostly two-dimensional, investigating infinite-height cylinders. Simulation of the vortex patterns and steady as well as unsteady loading at low Reynolds numbers was undertaken by Stansby et al. [10] using a vortex method. Recent efforts, employing different types of numerical simulation, include those of Mittal et al. [11] and Farrant et al. [12], also at relatively low values of Reynolds number. Furthermore, Papaioannou et al. [13] studied the three-dimensional effects in flow around two stationary cylinders with laminar and early turbulent regimes using a spectral/hp element method.

This paper presents results of Large Eddy Simulations (LES) of flow in an open channel over and around two submerged cylinders, one located downstream of the other. A high resolution grid is employed in the vicinity of the cylinders to obtain precise information on the zone around and between the cylinders. To the best of our knowledge, this is the first investigation of such a flow with the two finite-height cylinders arranged in tandem. 


\section{Configuration investigated}

The flow around two cylinders of finite height arranged in tandem, where one cylinder is located downstream of the other, studied here corresponds to an experiment carried out by Kadota et al. [4]. These authors performed measurements on various configurations of submerged two-in-tandem cylinders with a 2D Particle Image Velocimetry (PIV) system in a water tunnel. In their flume experiments, two cylinders of diameter $\mathrm{D}=20 \mathrm{~mm}$ and height $\mathrm{H}=50 \mathrm{~mm}$ were submerged under different water level heights $\mathrm{h}$ (in the present simulations we used $\mathrm{h}=60 \mathrm{~mm}$ ) and at a spacing $\mathrm{L}$ of $2 \mathrm{D}$. The Reynolds number, based on the cylinder diameter and the free-stream velocity $u_{\infty}$ is 1500 . Prior to the simulations, the experimenters provided the information that the approach flow was almost uniform, with a very thin boundary layer. For $\mathrm{L} / \mathrm{D}=2$, the flow corresponds to the quasi-steady reattachment regime where no vortex shedding occurs in the gap region.

The calculation domain spans $12.5 \mathrm{D}$ in streamwise, $6 \mathrm{D}$ in spanwise and 3D in vertical direction (i.e. the full water depth), respectively (Figure 1). The width and the height of the computational domain were selected to correspond to the flow cross section in the experiment. The origin of coordinates system was placed at the base of the first cylinder and $\mathrm{x}, \mathrm{y}, \mathrm{z}$ represent the streamwise, wallnormal and lateral direction, correspondingly. The grid consists of 516 x $256 \times$ 192 grid points in streamwise, spanwise and vertical directions, respectively, which sums to a total of approximately 25 Million grid points. The grid spacings in terms of wall units are the same in all directions, namely $\Delta x+=\Delta y+=\Delta z+\approx 1$ near the cylinder surface. Figure 2 shows the grid in a horizontal and a vertical plane indicating the refinement of the grid towards the cylinders (note that only every 5th grid line is plotted). The outflow is located at $x / D=9.5$ where a convective condition is applied. At the inflow $(\mathrm{x} / \mathrm{D}=-3.5)$ a constant velocity $\mathrm{u}=\mathrm{u}_{\infty}$ without fluctuations is specified. At the channel bed and lateral walls as well as the cylinder walls, the no-slip condition is used and the free surface is set as a frictionless rigid lid, treated by the slip condition.

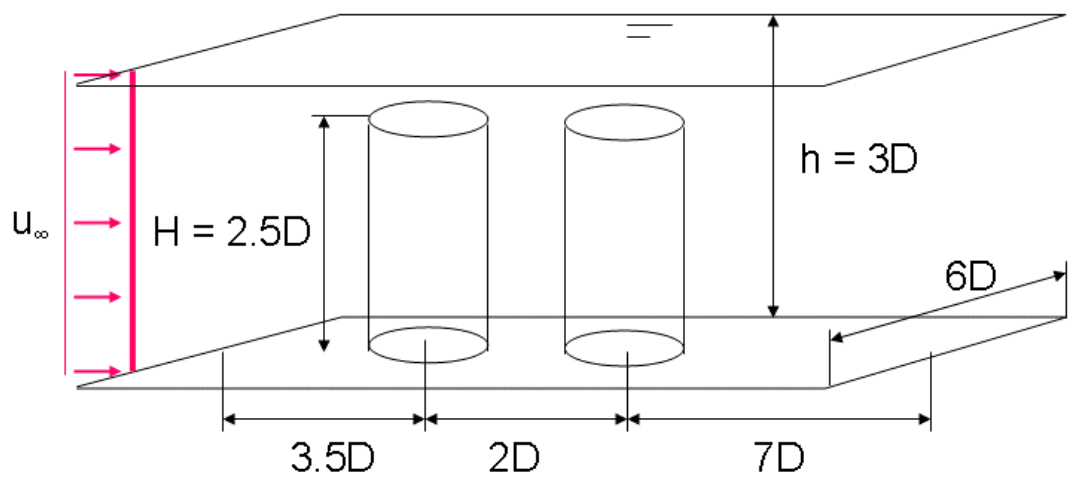

Figure 1. Schematic of the domain used in the simulation. All the dimensions are referred to the cylinder diameter. 

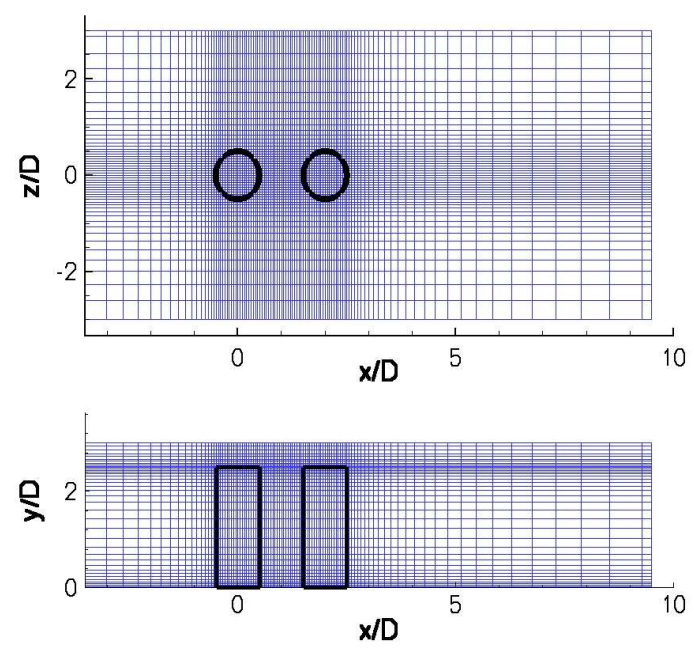

Figure 2. Two-dimensional cuts in wall-parallel direction (top) and wall-normal direction (bottom) through the computational grid. Only every 5th point is plotted.

\section{Numerical method and LES modelling}

The LES code MGLET, originally developed at the Institute for Fluid Mechanics at the Technical University of Munich [14] was used to perform the Large Eddy Simulations. The code solves the filtered Navier-Stokes equations discretised with the finite-volume method on a staggered Cartesian grid. Convective and diffusive fluxes are approximated with central differences of second order accuracy and time advancement is achieved by a second order, explicit AdamsBashford scheme. The Poisson equation for coupling the pressure to the velocity field is solved iteratively with the SIP method of Stone [15]. The subgrid-scale stresses appearing in the filtered Navier-Stokes equations are computed using the dynamic approach of Germano et al. [16]. The no-slip boundary condition is applied on the surface of the cylinders where the immersed boundary method is employed (Verzicco et al. [17]). This method is a combination of applying body forces in order to block the cells that are fully inside the cylinder and a Lagrangian interpolation scheme of third order accuracy, which is used for the cells that are intersected by the surfaces of the cylinders to maintain the no-slip condition.

\section{Results and Discussion}

The results will be presented in two stages. First, the instantaneous flow will be discussed by means of the structures visualized by an iso-surface of the pressure fluctuation within the flow. Secondly, the average flow will be described by comparison to the experiments performed by Kadota et al. [4]. 


\subsection{INSTANTANEOUS FLOW}

An impression of the complex flow structures can be obtained from Figure 3, where a snapshot of an iso-surface of the normalized instantaneous pressure fluctuation (obtained as the difference between the instantaneous and average pressure) is plotted. The following comments are based on this picture and other views and animations not shown in the present paper. Previous (Fröhlich and Rodi [18]) and ongoing research has yielded comprehensive information about the turbulent structures that occur in the flow past single finite-height cylinders of different height-to-diameter ratios (H/D) albeit at higher Reynolds numbers (in the range 22000-43000). In these cases, vortex shedding occurs near the ground plate and could be observed more clearly the larger the ratio H/D is. A pair of trailing vortices originating from the top is swept downstream, reducing the vortex shedding in the upper part of the cylinder. In the present case of two-intandem cylinders, a different flow occurs downstream of the first cylinder. Vortex shedding is suppressed by the presence of the second cylinder; rather the shear layers separating on both sides from the first cylinder reattach on the second cylinder and consequently only fairly small-scale structures are present in this region and no large-scale structures typical of shed vortices. In the far field downstream of the second cylinder, larger structures are present and increase in size, but considerably less so than in the case of the single cylinder simulated by Fröhlich and Rodi [18]. The processing of the results carried out so far did not give any clear evidence of von Karman vortices and further processing is under way to examine this.

\subsection{AVERAGE FLOW}

The average flow is now discussed, starting with what happens on the surface of both cylinders. Figure 4 presents the streamlines as seen from the side and from the rear on the surfaces on both cylinders. First of all, a considerable difference can be seen on the free end of the cylinders. On the top of the upstream cylinder, the flow separates at the front corner and reattaches at $\mathrm{x} / \mathrm{D} \approx 0.2$ (see Figure 5 ), leading to a re-circulation and reverse flow in the front part of the top as can be observed as well in Figure 4. This separation was not found in the experiments of Kadota et al. [4], probably due to a lack of resolution of the PIV measurements, but it has been reported in various single finite-height cylinder measurements [19-20]. In these also the corresponding pair of vertical vortices on the top surface have been observed by oil flow pictures and smoke visualization, which represent the spiral nodes of the well known owl-face configuration that can be seen in Figure 4. However, on the top of the second cylinder the flow is attached because the approach flow is more or less parallel to the top plane (see Figure 6) so that also the owl-face structure is absent and the surface streamlines are more or less parallel in the main flow direction. On the side surfaces, there is a clear 
nearly vertical separation line on the first cylinder, while the situation is more complex on the side of the second cylinder. In the gap between the cylinders, the trailing vortices originating from the top of the first cylinder are moved downwards and inward by the mean motion (see Figure 6) and impinge at about half height on the second cylinder leading to a nodal point of the separation line. Also, over most of the cylinder height, the motion is downward in the rear part of the first cylinder, only near the top it is upward as the flow separates at the trailing edge and forms a small recirculation region shown in Figure 5. In contrast, the flow in the rear of the second cylinder is directed upwards from the bottom to the very top, as is clear also from Figure 6.
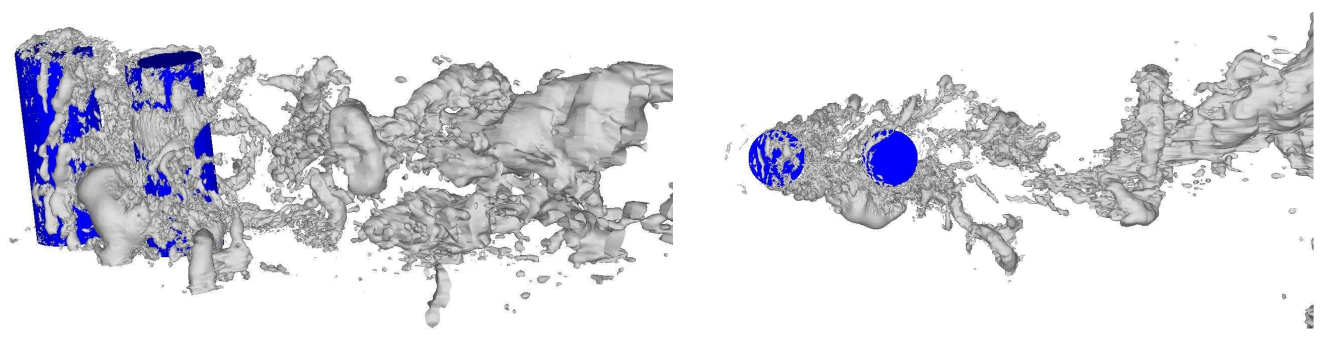

Figure 3. Instantaneous flow structures obtained by the pressure fluctuation $\left(p^{\prime}=0.05\right)$ viewed from an oblique angle from the rear (left) and from the top (right).

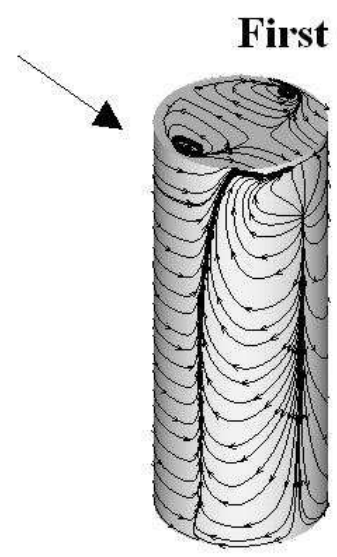

side view
First Cylinder

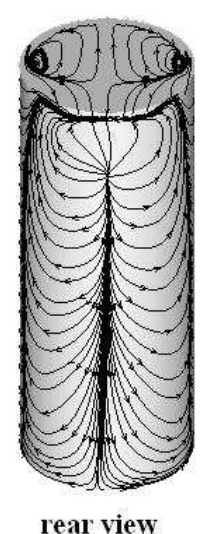

Second Cylinder

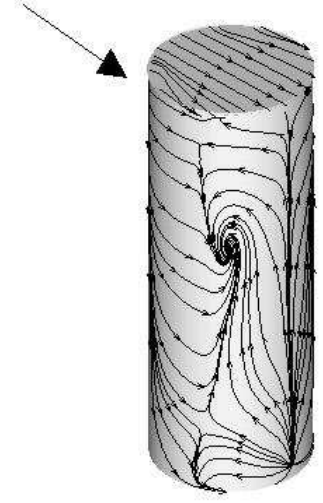

side view

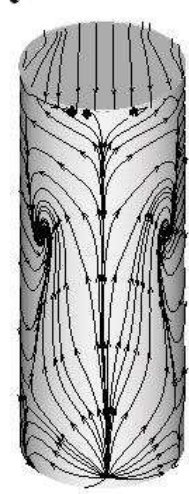

rear view

Figure 4. Surface streamlines from both cylinders. In either case, left is the oblique view while right is the rear view.

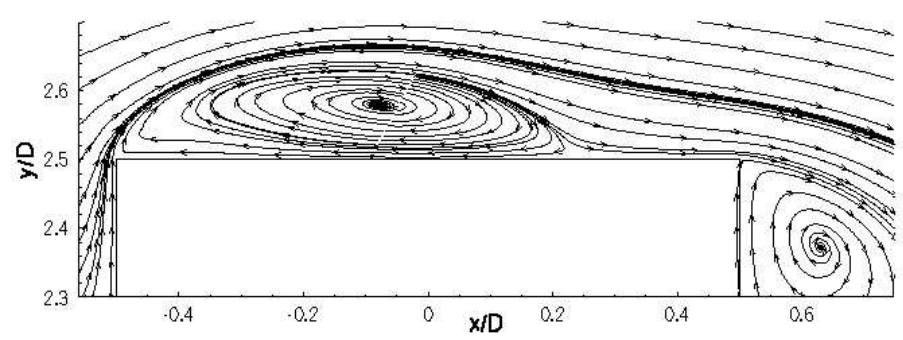

Figure 5. Close-up of the separation over the free end of the upstream cylinder, showing a large recirculation. 
Now comparisons between the LES results obtained with the MGLET code and the experimental data from Kadota et al [4] are discussed. Figure 6 compares the average streamlines and contours of the u-velocity component in the symmetry plane (top of the figure) and a wall-parallel plane (bottom of the figure) located near the mid-height of the cylinders $(y / D=1.5)$. On the whole, there is fairly good correspondence between calculations and measurements, but some differences can be noticed. It was mentioned already that in the experiment no separation was detected on top of the first cylinder. Further, the experiments show a small recirculation region at the foot in front of the second cylinder. There is a tendency towards development of such a recirculation region, but there is no actual re-circulation. The difference may be due to some differences in the approach-flow velocity as the development of the flow in this region is rather sensitive to this. The authors were only recently informed by the experimenters that the approach flow was not entirely uniform and had a thicker boundary layer than initially stipulated.

As can be seen from Figure 6, the gap between the cylinders is entirely filled by the pair of recirculation zones and there is a fairly strong downward flow almost everywhere in the gap, bringing in fluid from the top and moving it outwards around the second cylinder near the bottom. The flow behind the second cylinder is very different but also quite different from that behind a single cylinder. In the centre plane there is a detached core of circulation just below the mid-height at which fluid rushes in from the sides to fill the wake at this height, where the recirculation zones are quite small (see Figure 6), much smaller than in the single cylinder case. It appears that in this region with particularly complex flow the simulation is not yet fully converged as some asymmetry of average flow quantities has noticed (see Figure 8). Above, an upward motion is caused behind the cylinder by the fairly high velocity of the attached flow over the top in entraining fluid. Below mid-height there is an almost closed recirculation with the separation region in horizontal planes reaching further downstream, closer to what was observed behind the single cylinder. All this is in fairly good agreement with the experimental results, as can be seen from Figure 6. The complex flow behaviour can also be seen from the vertical average u-velocity profile in the symmetry plane at $\mathrm{x} / \mathrm{D}=3$ presented in Figure 7 , and also here there is reasonable agreement between the calculations and the measurement. There are larger deviations for the velocity profile at $\mathrm{x} / \mathrm{D}=1$ in the middle of the gap between the cylinders, also shown in Figure 7. Here, the negative velocity at mid-height is over-predicted. This may again be due to the specified approach flow velocity being somewhat different from the one in the experiment, but it may also be partly caused by the assumption of a rigid lid which may not be fully justifiable in this case where there is a fairly small distance between the top of the cylinders and the water surface and this may cause non-negligible surface elevations over the cylinders, particularly the first one. 

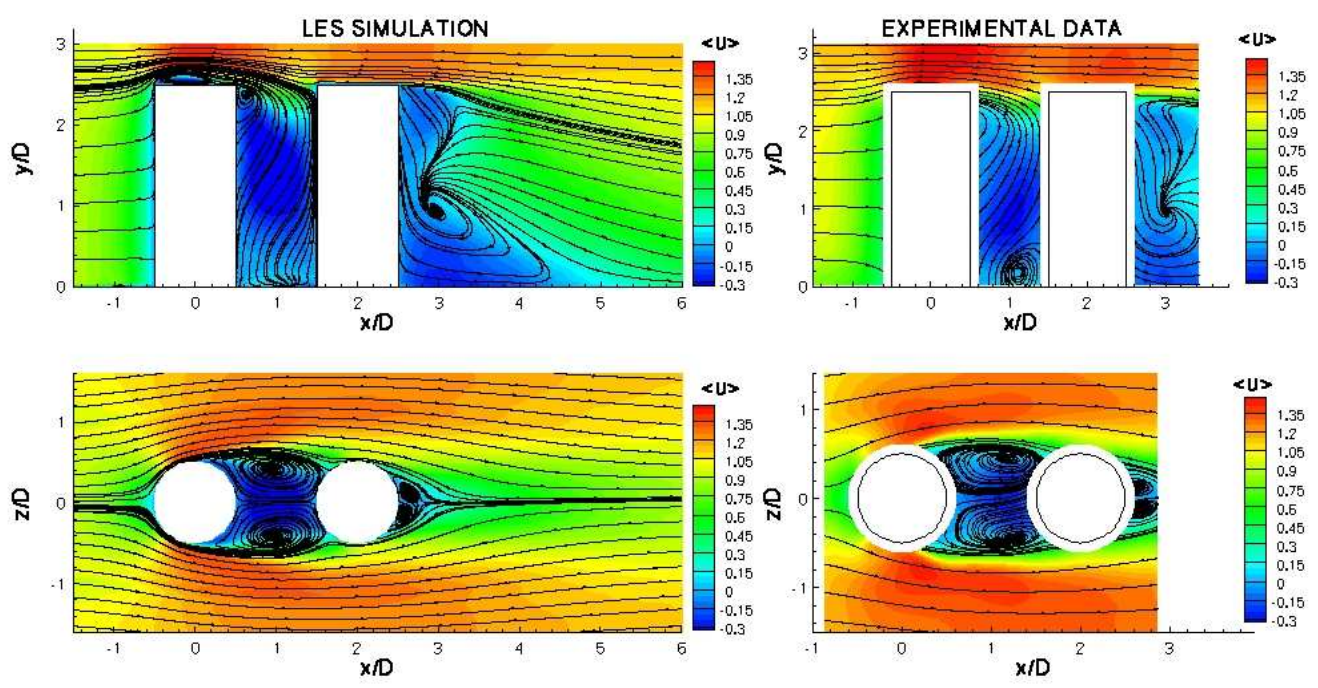

Figure 6. Average streamlines in the center plane (top) and near the mid height of the cylinders (bottom). Left: Large Eddy Simulation results, right: experiment.
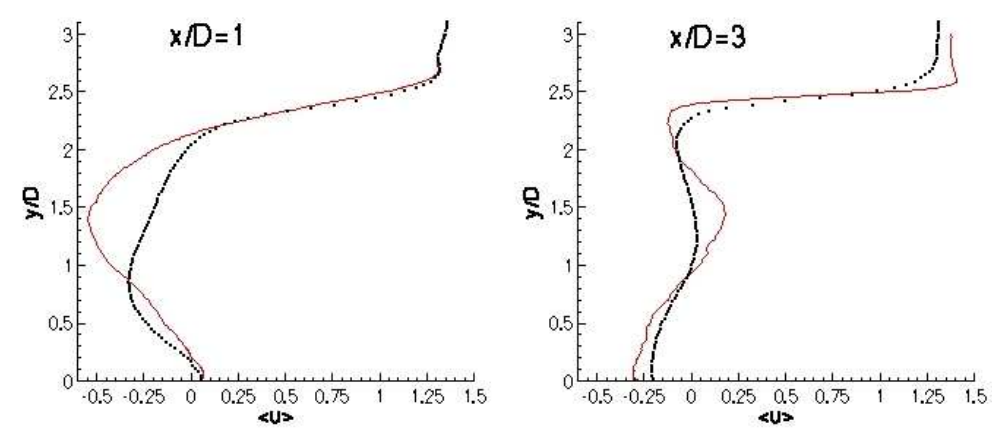

Figure 7. Streamwise mean velocity in wall-normal direction at $\mathrm{x} / \mathrm{D}$ equal to 1 (left) and 3 (right). - LES, - Experiments of Kadota et al. [4].

Figure 8 represents the secondary motion at various cross sections and allows us to follow the trailing vortices produced on the top of the upstream cylinder. In the first plane, located at the middle of the first cylinder, the formation of the two trailing vortices can be observed, similar to the structures obtained by smoke visualization by Roh and Park [19]. These trailing vortices are moved downstream and downward by the mean motion, and in the middle of the gap between both cylinders their centers are located at $\mathrm{y} / \mathrm{D} \approx 1.5$ (similar values were obtained by Fröhlich and Rodi [18] for a single cylinder with the same ratio $\mathrm{H} / \mathrm{D}=2.5$ ). These vortices then impinge on the second cylinder causing a secondary motion near the cylinder surface at a height of $\mathrm{y} / \mathrm{D}=0.5$ and move further downward past this cylinder and merge with the horse-shoe vortex that is likely to be present there (see last plane at $\mathrm{x} / \mathrm{D}=4$ ). On top of the second cylinder, two further very weak vortices are produced, but these decay quickly and are not visible in the planes downstream. 


\section{Conclusions}

We have presented in this paper results of a Large Eddy Simulation of the flow around two cylinders arranged in tandem where one cylinder is located downstream of the other. The instantaneous and average flow reveals that the flow belongs to the reattachment regime; hence, as expected, no shedding vortices are formed in the gap between cylinders.

The flow behaviour is different from a single cylinder flow because the second cylinder perturbs the formation of a von Karman vortex sheet and the trailing vortices formed on the top of the first cylinder.

Comparison with experimental data shows generally good agreement, although some differences are observed in the gap between the cylinders that could be due to some differences in the approach flow velocity and the small submergence of the cylinders possibly causing changes in the water surface level above the upstream cylinder that was not taken into account in the LES.
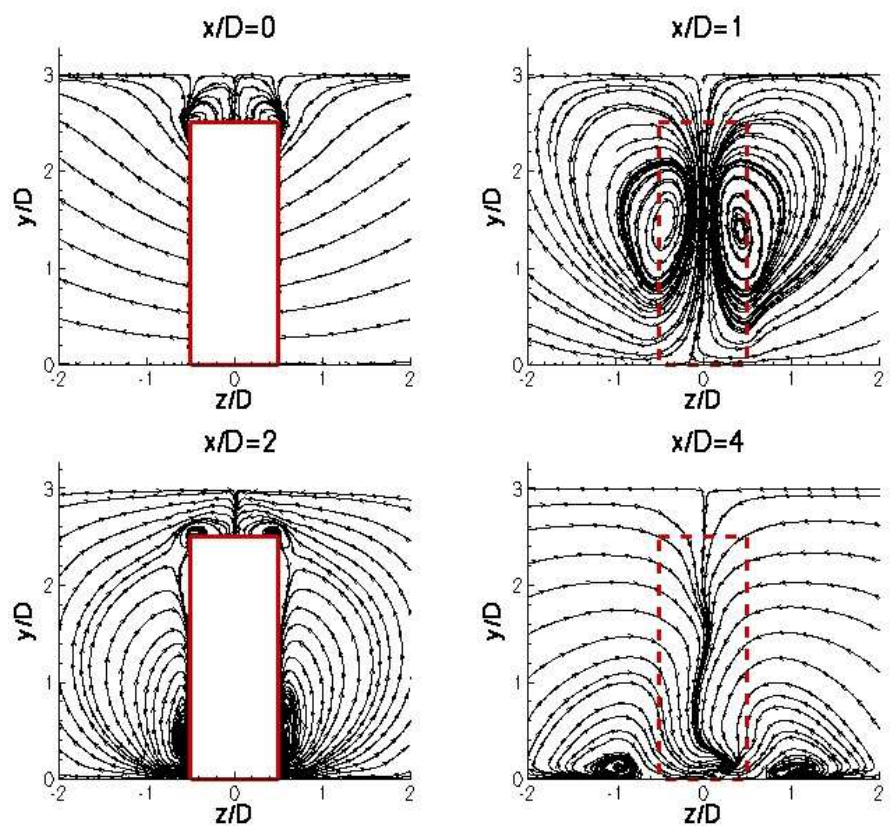

Figure 8. Streamlines in planes normal to the x-axis at the middle of each cylinder, between them and downstream the second one.

\section{Acknowledgements}

The authors acknowledge funding by the German Research Foundation and the support of the first author by the Politechnic University of Valencia. The authors would like to thank the steering committee of the Bavarian High Performance Computing Centre (HLRB) in Munich for granting computing time on the SGI Altix 4700. The authors also thank Dr. Kadota for providing his experimental data. 


\section{References}

1. Iragashi, T., Characteristics of the flow around two circular cylinders arranged in tandem. Bulletin of JSME. 24 (1981) 323-331.

2. Zdravkovich, M. M., The effect of interference between circular cylinders in cross flow. $J$. Fluids Struct. 1 (1987) 239-261.

3. Luo, S.C., Gan, T.L. and Chew, T.S., Uniform flow past one (or two in tandem) finite length circular cylinder(s). J. Wind Eng. Ind. Aerodyn. 59 (1996) 69-93.

4. Kadota, A., Aragao, R. and Suzuki, K., Visualization of flow pattern around two-in-tandem cylinders. Personal communication (2007).

5. Fischer Antze, T., Stoesser, T., Bates, P. B. and Olsen, N.R., 3D numerical modelling of open-channel flow with submerged vegetation. IAHR J. Hyd. Res. 39 (2001) 303-310.

6. Ljungkrona, L., Norberg, C.H. and Sundén, B., Free-Stream turbulence abd tube spacing effects on surface pressure fluctuations for two tubes in an in-line arrangement. J. Fluids Struct. 5 (1991) 701-727.

7. Oka, S., Kostic, Z.G. and Sikmanovic, S., Investigation of the heat transfer process in tube banks in cross flow. In: International Seminar on Recent Development in Heat Exchangers. Trogir, Yugoslavia (1972).

8. Tanida, Y., Okajima, A. and Watanabe, Y., Stability of a circular-cylinder oscillating in uniform flow or in a wake. J. Fluid Mech. 61 (1973) 769-784.

9. Summer, D., Price, S. J. and Paidossis, M. J., Tandem cylinders in impulsively started flow. J. Fluids Struct. 13 (1999) 955-965.

10. Stansby, P.K., Smith, P. A. and Penoyre, R., Flow around multiple cylinders by the vortex method. In: Proceedings International Conference on Flow Induced Vibration. England, Cranfield. (1987) 41-50.

11. Mittal, S., Kumar, V. and Raghuvanshi, A., Unsteady incompressible flows past two cylinders in tandem and staggered arrangements. Int. J. Numer. Meth. Fluids 25 (1997) 1315-1344.

12. Farrant, T., Tan, M. and Price, W.G., A cell boundary element method applied to laminar vortex-shedding from arrays of cylinders in various arrangements. J. Fluids Struct. 14 (2000) 375-402.

13. Papaioannou, G. V., Yue, D., Triantafyllou, M. and Karniadakis, G., Three-dimensionally effects in flow around two tandem cylinders. J. Fluid Mech. 558 (2006) 387-413.

14. Tremblay, F. and Friedrich, R., An algorithm to treat flows bounded by arbitrarily shaped surfaces with Cartesian meshes. In: Notes on Numerical Fluid Mechanics. Ed. Springer 77 (2001).

15. Stone, H.L., Iterative solution of implicit approximation of multi-dimensional partial differential equations. SIAM J. Numerical Analysis 3 (1968).

16. Germano, M., Piomelli, U., Moin, P. and Cabot, W.T., A dynamic subgrid-scale eddy viscosity model. Phys. Fluids 3 (1991) 1760-1765.

17. Verzicco, R., Mohd-Yusof, J., Orlandi, P. and Haworth, D., Large Eddy Simulation in Complex Geometric Configurations Using Boundary Body Forces. AAIA J. 38 (2000) 427433.

18. Fröhlich, J. and Rodi, W., LES of the flow around a circular cylinder of finite height. Int. J. Heat Fluid Flow 25 (2004) 537-548.

19. Roh, S.C. and Park, S.O., Vortical flow over the free end surface of a finite circular cylinder mounted on a flat plate. Exp. Fluids. 34 (2003) 63-67.

20. Delery, J.M., Physics of vortical flows. J. Aircraft. 29 (1992) 856-876. 